\title{
Fibrina rica em plaquetas e sua utilização na cirurgia de levantamento de assoalho de seio maxilar
}

\author{
Rich in fibrina in platelets used in maxillary sinus floor augmentation surgery \\ Rico em fibrina em plaquetas y su uso em cirugía para elevación del piso maxilar
}

Recebido: 04/06/2021 | Revisado: 14/06/2021 | Aceito: 18/06/2021 | Publicado: 02/07/2021

\author{
Mariany Gonçalves Pucetti \\ ORCID: https://orcid.org/0000-0003-0251-9765 \\ Universidade Federal de Juiz de Fora, Brasil \\ E-mail: mariany_jf@hotmail.com \\ Júlia de Souza Faria \\ ORCID: https://orcid.org/0000-0002-8903-9766 \\ Universidade Federal de Juiz de Fora, Brasil \\ E-mail: juliadesouzafaria99@gmail.com \\ Rafaela Henriques Moreira \\ ORCID: https://orcid.org/0000-0002-8388-7414 \\ Universidade Federal de Juiz de Fora, Brasil \\ E-mail: rafaela_moreira_18@hotmail.com \\ Vitória de Assis Manso Neves \\ ORCID: https://orcid.org/0000-0002-8810-9670 \\ Universidade Federal de Juiz de Fora, Brasil \\ E-mail: vitoriaassisneves@hotmail.com \\ Adriano de Oliveira Loures \\ ORCID: https://orcid.org/0000-0002-0598-5377 \\ Universidade Federal de Juiz de Fora, Brasil \\ E-mail: adrianoloures@outlook.com \\ Breno Nogueira Silva \\ ORCID: https://orcid.org/0000-0001-5655-4684 \\ Universidade Federal de Juiz de Fora, Brasil \\ E-mail: brenosrj@terra.com.br \\ Neuza Maria Souza Picorelli Assis \\ ORCID: https://orcid.org/0000-0002-8612-5044 \\ Universidade Federal de Juiz de Fora, Brasil \\ E-mail: neuzapicorelli@ terra.com.br
}

\begin{abstract}
Resumo
Os concentrados de Plaquetas (CPs), como a Fibrina Rica em Plaquetas (PRF) são utilizados em Odontologia com o intuito de melhorar a cicatrização. Um dos procedimentos cirúrgicos onde este material é mais utilizado é na cirurgia de elevação do assoalho sinusal (EAS). Pode auxiliar tanto a regeneração de tecidos duros quanto moles e pode ser utilizado sozinho ou com outros biomateriais e auxiliar na prevenção e tratamento da perfuração da membrana de Schneider. O objetivo deste estudo foi revisar a literatura sobre a PRF e suas aplicações na cirurgia de levantamento de assoalho de seio maxilar. Foi realizado uma busca de artigos, na língua inglesa, utilizando as bases de dados LILACS, Pubmed e Google Scholar, no período de 2006 a 2021. Os artigos científicos incluíram estudos clínicos, revisões de literatura e revisões sistemáticas. A PRF se mostrou um método fácil, simples e econômico de auxílio na reparação tecidual. Pode auxiliar na correção de deficiências de altura óssea, complementando e auxiliando positivamente a cirurgia de levantamento de assoalho sinusal. No entanto, ainda não existe um consenso em relação à sua contribuição efetiva na cirurgia de levantamento de seio. Assim, mais estudos randomizados e utilizando o mesmo protocolo de obtenção deste concentrado de plaquetas são necessários.
\end{abstract}

Palavras-chave: Fibrina rica em plaquetas; Levantamento do assoalho do seio maxilar; Regeneração tecidual guiada.

\begin{abstract}
Platelet Concentrates (PCs), such as Platelet Rich Fibrin (PRF), are used to improve wound healing. One of the surgical procedures where this material is most used is in maxillary sinus floor augmentation surgery. PRF can assist the regeneration of hard and soft tissues, be used alone or with other biomaterials and prevent and treat Schneider membrane perforation. The aim of this study was to review the literature on PRF and its applications in maxillary sinus floor surgery. A search for articles was carried out, in English, using a LILACS, Pubmed e Google Scholar databases, from 2006 to 2021. Clinical trials, literature reviews and systematic reviews were included. PRF proved to be an easy, simple and economical method of assisting tissue repair. It can assist in the correction of bone height deficiencies, complementing and positively assisting maxillary sinus floor augmentation surgery. However, there is a lack of
\end{abstract}


consensus regarding the effective contribution of PRF to maxillary sinus floor augmentation surgery. Thus, more randomized studies using the same protocol for obtaining this platelet concentrate are needed.

Keywords: Platelet-rich fibrin; Sinus floor augmentation; Guided tissue regeneration.

\section{Resumen}

Los concentrados de plaquetas (CP), como la fibrina rica en plaquetas (PRF), se utilizan en odontología para mejorar la cicatrización. Uno de los procedimientos quirúrgicos donde más se utiliza este material es en la cirugía de elevación del suelo del seno (ESS). Puede ayudar tanto a la regeneración de tejidos duros como blandos y puede usarse solo o con otros biomateriales y ayudar en la prevención y el tratamiento de la perforación de la membrana de Schneider. El objetivo de este estudio fue revisar la literatura sobre la PRF y sus aplicaciones en la cirugía de levantamiento del piso del seno maxilar. Se realizó una búsqueda de artículos en idioma inglés, utilizando la bases de datos LILACS, Pubmed e Google Scholar, de 2006 a 2021. Los artículos científicos incluyeron estudios clínicos, revisiones de la literatura y revisiones sistemáticas. PRF demostró ser un método fácil, simple y económico para ayudar a la reparación de tejidos. Puede ayudar en la corrección de las deficiencias de altura ósea, complementando y asistiendo positivamente a la cirugía de elevación del piso del seno. Sin embargo, todavía no hay consenso sobre su contribución efectiva a la cirugía de levantamiento de senos. Por lo tanto, se necesitan más estudios aleatorizados que utilicen el mismo protocolo para obtener este concentrado de plaquetas.

Palabras clave: Fibrina rica en plaquetas; Elevación del piso del seno maxilar; Regeneración tisular dirigida.

\section{Introdução}

A coagulação sanguínea cria conexões biológicas baseadas em fibrina dentro de um tecido ferido ou na interface entre os tecidos. Seguindo esse princípio, foram criadas as colas de fibrina e os concentrados de plaquetas (CPs), como a fibrina rica em plaquetas (PRF): uma ferramenta polimórfica e adaptativa que é um excelente adjuvante cirúrgico, reproduzindo o processo natural de coagulação. Devido a sua eficiência, cresce cada vez mais o seu uso em Odontologia. Esses materiais foram projetados para melhorar tanto a cicatrização, quanto a angiogênese no local da lesão e podem ser usados sem efeitos adversos significativos na maioria dos tecidos. Além disso, apesar da natureza intrínseca, a cirurgia oral e maxilofacial requer volumes relativamente pequenos de produtos e a necessidade de estimular a cicatrização é muito importante (Del Corso et al., 2012).

A PRF é a próxima geração de CPs equipados para melhorar a cicatrização sem manipulação bioquímica do sangue, considerado uma evolução do adesivo de fibrina (Kumar et al., 2016). É um aditivo biológico cirúrgico preparado por manipulação de sangue autólogo (Shah, Triveni, Thomas \& Singh, 2017), usado na regeneração de tecidos moles e tecidos ósseos (Ghanaati et al., 2018). Dessa forma, por ser utilizado em pequenas quantidades e com diversas aplicabilidades, a PRF desenvolveu-se amplamente em cirurgia oral e maxilofacial (Del Corso et al., 2012) especialmente pela relevância de ser uma técnica simples, rápida e econômica obtendo resultados favoráveis (Del Corso et al., 2012; Ortega-Mejia et al., 2020; Simonpieri et al., 2012).

A inadequação da altura óssea residual (AOR) devido à pneumatização sinusal e à remodelação óssea alveolar pode comprometer a opção de reabilitação suportada por implante em maxilas posteriores atrofiadas (Dominiak, S., KarugaKuźniewska, E., Popecki, P., \& Kubasiewicz-Ross, P. 2021; Testori et al., 2019). Para resolver este problema e promover uma reabilitação adequada do paciente é sugerido o levantamento do assoalho do seio maxilar, onde é feito um procedimento cirúrgico que aumenta a quantidade de osso na maxila posterior pela elevação da membrana do seio, denominada membrana de Schneider. O objetivo dessa cirurgia é obter osso para apoiar um ou mais implantes dentários que podem ser instalados ao mesmo tempo que a cirurgia sinusal (colocação simultânea) ou após um período de cicatrização (colocação tardia). A escolha da técnica cirúrgica depende da quantidade e qualidade do osso na crista alveolar já que a restauração bem-sucedida do implante é altamente dependente de volume e densidade óssea suficientes (Liu et al., 2019).

A EAS é um dos procedimentos mais comuns de enxerto ósseo em Implantodontia e, por isso, tornou-se um dos procedimentos mais investigados com CPs. Além disso, é um excelente modelo de cicatrização para a avaliação da remodelação óssea, por ser uma cavidade fechada e protegida, onde as interferências no ambiente oral e nas funções maxilomandibulares são 
mínimas. Assim, a cicatrização óssea pode, portanto, ser avaliada com menor interferência de fatores externos (Simonpieri et al., 2012).

No entanto, a elevação tradicional do assoalho do seio maxilar pode apresentar sérias complicações pós-operatórias e longos períodos de cicatrização para pacientes com AOR insuficiente. A PRF pode promover a cicatrização dos tecidos e prevenir a perfuração da membrana (Shah et al., 2017; Ortega-Mejia et al., 2020; Wang et al., 2019). Segundo Barbu, Andreescu, Comaneanu, Referendaru, e Mijiritsky (2018), a associação do enxerto ósseo bovino particulado com a PRF pode permitir uma cicatrização mais rápida e reabilitação precoce. Os vários componentes da matriz, como a fibronectina, vitronectina, glicosaminoglicanos e a própria fibrina, e os fatores de crescimento dos concentrados de plaquetas estimulam logicamente o "reinício" das células enxertadas, sua rápida proliferação e/ou diferenciação e a reconstrução e remodelação das células enxertadas no tecido (Del Corso et al., 2012).

Ainda assim, é importante ressaltar que, seja qual for o produto, o uso bem-sucedido dessas preparações ricas em fatores de crescimento depende da habilidade dos cirurgiões-dentistas que precisam compreender a preparação, uso e a combinação correta das tecnologias para obter os melhores resultados possíveis na reabilitação dos pacientes. Diante disso, percebe-se que o uso da PRF é promissor e deve ser avaliado se há vantagem clínica de sua utilização na cirurgia de levantamento do assoalho de seio maxilar.

\section{Metodologia}

Trata-se de uma revisão de literatura. A busca dos artigos foi realizada nas bases de dados LILACS, Pubmed, Scopus e Google Scholar, com as palavras-chave "Platelet-Rich Fibrin", “Sinus Floor Augmentation" e "Guided Tissue Regeneration". Foram considerados elegíveis estudos em inglês publicados entre 01/2006 a 05/2021 e relacionados à PRF e cirurgias de levantamento de assoalho de seio maxilar, de acordo com os títulos e resumos dos artigos. Foram excluídos artigos na qual o resumo apresentava acesso restrito, resumo com acesso disponível e artigo restrito, além de comentários, editoriais, cartas, diretrizes das sociedades médicas e artigos que não apresentaram relação com o tema do manuscrito. As referências bibliográficas dos artigos incluídos também foram consultadas, em busca de outros estudos que possam contemplar os objetivos dessa revisão de literatura.

\section{Discussão}

O processo natural de cicatrização em qualquer ferida começa com a coagulação do sangue levando à formação de fibrina/coágulo de plaquetas e matriz. A cicatrização dos tecidos duros e moles é realizada por vários eventos intra e extracelulares, que são mediados por sinais de proteínas (Kumar et al., 2016). Plaquetas, leucócitos, fibrina e fatores de crescimento são os principais fatores ativos no processo de cicatrização de feridas (Agrawal, 2017). As plaquetas estão envolvidas nesse processo por meio da formação de coágulos sanguíneos e da liberação de fatores de crescimento, que promovem e mantém o processo (Kumar et al., 2016; Agrawal, 2017). Este processo ocorre após a lesão tecidual e pela exposição do endotélio vascular com a liberação de fatores de crescimento, dentre os quais se encontram o Fator de Crescimento Derivado de Plaquetas, Fator de Crescimento Epitelial, Fator de Crescimento Fibroblástico Básico, Fator de Crescimento Semelhante a Insulina, Fator de Crescimento Endotelial Vascular e Fator de Crescimento Transformador $\beta$. Assim, as plaquetas no local da ferida definem o ritmo para o reparo, influenciando a chegada de outras células. (Agrawal, 2017). Os concentrados de plaquetas (CPs), em destaque a PRF, foram introduzidos para reforçar este processo natural de cicatrização de feridas, acelerando o processo de revascularização e migração de células (Agrawal, 2017; Ortega-Mejia et al., 2020).

A PRF foi desenvolvida na França pelo Dr. Choukroun e colaboradores para uso específico em cirurgia bucomaxilofacial, pioneiramente no uso da L-PRF para promoção da regeneração óssea relacionada aos implantes dentários. É 
um concentrado de plaquetas preparado a partir do próprio sangue do paciente, sem anticoagulante ou qualquer modificação bioquímica. É uma malha de fibrina, que pode servir como uma película reabsorvível, na qual citocinas plaquetárias, fatores de crescimento e células são aprisionadas e liberadas após um período de tempo e contém todos os componentes de uma amostra de sangue que são favoráveis para o processo de cicatrização (Choukroun et al., 2006; Kumar et al., 2016; Simonpieri et al., 2012). Por isso, o coágulo L-PRF, ou seja, leucócito e PRF, era conhecido como um "coágulo de sangue otimizado" (Agrawal, 2017). É considerado como uma manipulação tecidual através da transformação do sangue em um biomaterial natural para ser aplicado em tecidos (Simonpieri et al., 2012) com o objetivo de promover a regeneração tecidual (Agrawal, 2017; Kumar et al., 2016).

O sucesso dessa técnica depende principalmente do intervalo de tempo desde a coleta do sangue até sua transferência para a centrifugação, que deve ser feita em menor tempo possível (Kumar et al., 2016). No entanto, a técnica de preparação, tempo de espera, processo de transferência, temperatura da centrífuga e vibração são fatores que podem justificar alguns resultados heterogêneos encontrados (Agrawal, 2017). Além da presença ou ausência de leucócitos, há outros parâmetros que devem ser levados em consideração como a quantidade ou taxa de coleta de plaquetas, composição celular e preservação durante a coleta e transporte. Os parâmetros específicos da centrífuga usada também são importantes, tais como seu tamanho, vibração e a duração da centrifugação (Agrawal, 2017). Assim, a padronização do protocolo é necessária para se obter resultados reproduzíveis (Castro et al., 2017).

O protocolo PRF é simples: uma amostra de sangue é obtida sem anticoagulante em tubos de 10ml que são centrifugados a 3000 rpm (aproximadamente 400 g) por 10 minutos (Aoki et al., 2018; Barbu, Andreescu, Comaneanu, Referendaru, \& Mijiritsky, 2018; Kumar et al., 2018; Wang et al., 2019). A preparação da L-PRF requer uma centrífuga adequada e um kit de colheita que inclui: uma scalp (tipo butterfly) de calibre 24, e tubos de ensaio de $10 \mathrm{ml}$ para colheita do sangue. De acordo com a revisão sistemática de Ali, Bakry e Abd-Elhakam (2015), estudos demonstraram diferentes durações centrífugas, variando de 10 a 14 minutos (Ali, Bakry \& Abd-Elhakam, 2015). Isso pôde ser observado no estudo de Toffler, Toscano e Hotzclaw (2010) que realizaram a centrifugação por $12 \mathrm{~min}$ a $2700 \mathrm{rpm}$. Diversas modificações do protocolo convencional foram realizadas desde a sua criação a partir de pesquisas aprofundadas sobre suas aplicações clínicas. Já no protocolo proposto por Ghanaati em 2014, obtém-se fibrina rica em plaquetas avançada (A-PRF), em 1300 RPM durante 14 min, em um tubo patenteado. Em 2016, foi proposto um protocolo por Rujioka-Kobayashi e Miron em que se obtinha fibrina rica em plaquetas avançada + (A-PRF +) com 1300 RPM em um tempo de 8 min, usando tubos igual a A-PRF. Em 2015, Mourão propôs um protocolo para obtenção de fibrina rica em plaquetas injetável (i-PRF) onde é necessário 700 RPM, em 3 min em tubos não revestidos (apud Shah et al., 2017).

Em poucos minutos, a ausência do anticoagulante induz a ativação das plaquetas contidas na amostra, desencadeando uma cascata de coagulação; assim o sangue começa a coagular imediatamente com o simples contato com as paredes do tubo. $\mathrm{O}$ mecanismo envolvido é o fibrinogênio concentrado na parte superior do tubo que se combina com a trombina circulante quando centrifugado para formar fibrina. Após a centrifugação, o material se estabelece em três camadas, a camada de base dos glóbulos vermelhos, a camada superior do plasma acelular e o coágulo de PRF ao centro, contendo a maioria das plaquetas e leucócitos e plasma rico em plaquetas (Castro et al., 2017; Kumar et al., 2016; Toffler, Toscano \& Holtzclaw, 2010; Wang et al., 2019). Os coágulos são, então, colocados em uma grade na caixa PRF e comprimidos por uma tampa (esmagador), para criar uma membrana de fibrina. Alternativamente, os coágulos também podem ser colocados em cilindros contidos na caixa e comprimidos por pistões para criar um tampão de fibrina. Esses plugues de PRF, em certos casos, são preferidos às membranas porque são mais simples de inserir, comprimir e serem deslocados apicalmente na osteotomia preparada (Castro et al., 2017; Testori et al., 2019). Além disso, a PRF pode ser misturada a substitutos ósseos particulados, obtendo uma mistura fácil de manipular e usar como material de enxerto (Barbu et al., 2018). 
A PRF como aditivo cirúrgico biológico tem sido utilizada com sucesso em diversas aplicações na Odontologia (Shah et al., 2017). Destacam-se o seu uso durante a colocação de implante, particularmente estimulando a osseointegração, no tratamento de defeitos ósseos peri-implantares, na cirurgia reconstrutiva maxilofacial para o tratamento de pacientes em uso de bisfosfonatos e anticoagulantes, com maxila irradiada pós tratamento tumoral e procedimentos de levantamento sinusal (Shah et al., 2017; Simonpieri et al., 2012). Para optar pelo uso ou não da PRF, deve ser levado em consideração o custo envolvido, a forma, o volume do produto final (Agrawal, 2017), facilidade de aplicação e rapidez de preparação (Del Corso et al., 2012; Simonpieri et al., 2012, Kumar et al., 2016, Shah et al., 2017), além da liberação prolongada de fatores de crescimento (Shah et al., 2017). Como é utilizado o próprio sangue do paciente para a obtenção da PRF, a transmissão de doenças é eliminada (Kumar et al., 2016; Shah et al., 2017).

A PRF atua como um conector biológico entre os diferentes elementos do enxerto, captura células-tronco e, com o aprisionamento de citocinas, promove uma rápida migração celular, inclusive de células osteoprogenitoras ao mesmo tempo que possui efeito inibidor sobre osteoclastos (Kumar et al., 2016; Shah et al., 2017). Além disso, atua como uma matriz que suporta a neoangiogênese e desempenha um importante papel na revascularização do tecido (Choukroun et al., 2006; Kumar et al., 2016). Promove a mitose das células, mediada pelo Fator de Transformação do Crescimento $\beta$ (fibroblastos, células-tronco da medula, células endotelias, pré-osteoblastos e células mesenquimais), possui efeitos imunomoduladores mediados pela fibrina e seus produtos de degradação, fibronectina, leucócitos e interleucina 4 (Shah et al., 2017). Assim, a cobertura do defeito ósseo enxertado com um gel de fibrina necessariamente promove a cicatrização dos tecidos moles e a proteção do local cirúrgico, levando à aceleração do fechamento da ferida e à regeneração (Ali et al., 2015; Del Corso et al., 2012).

A perda de osso na maxila posterior, resultante principalmente da reabsorção óssea alveolar que pode ser decorrente de vários fatores como a perda dentária, doença periodontal, traumatismo, extrusão dentária, causa a pneumatização do seio maxilar e dificulta o tratamento com implantes dentários. Esse problema pode ser superado com o aumento da altura alveolar por meio de elevação do seio maxilar, seguido do enxerto (Dominiak et al, 2021; Liu et al., 2019). O que define a técnica de levantamento de seio maxilar são a quantidade e a qualidade de osso alveolar remanescente, e são indicadas algumas modalidades cirúrgicas, como o Acesso Lateral ou Caldwell-Luc (Ali et al., 2015; Aoki et al., 2018; Castro et al., 2017) e o levantamento atraumático com osteótomos (Aoki et al., 2018; Narang et al., 2015; Toffler et al., 2010). A técnica de osteótomo, de acordo com Narang et al. (2015), é mais previsível com a colocação simultânea do implante, quando há menos que 5 a 7 mm de altura do osso alveolar preexistente abaixo do seio. Em alguns procedimentos, pode-se usar o endoscópio, dando maior precisão às cirurgias (Liu et al., 2018; Wang et al., 2019) ou até a piezocirurgia (Barbu et al., 2018; Testori et al., 2019). O enxerto ósseo pode dar resultados satisfatórios na cicatrização óssea, mas não tem impacto na cicatrização de tecidos moles e pode ser comprometido por infecções e inflamações locais (Simonpieri et al., 2012). A cirurgia de elevação de seio maxilar é um procedimento que possibilita uma boa avaliação da cicatrização e remodelação óssea (Simonpieri et al., 2012) e fácil para investigar os numerosos materiais de enxerto utilizados e os fatores adjuvantes, como a PRF (Molemans et al., 2019; Ocak et al., 2017). Esse material reduz o período de cicatrização e amplia as indicações do tratamento da elevação do seio transcretal (Liu et al., 2018).

As membranas de L-PRF promovem excelentes resultados clínicos (Del Corso et al., 2012), uma vez que protegem o sítio cirúrgico e promovem a cicatrização dos tecidos moles (Kumar et al., 2016). Efeitos benéficos na regeneração óssea e na cirurgia de implante são sugeridos quando este material é aplicado (Castro et al., 2017; Dominiak et al., 2021). Ghanaati et al., 2018). A elevação do assoalho do seio apenas com PRF é um procedimento seguro porque ela é capaz de proteger a membrana do seio maxilar durante a instalação do implante e, assim, evita sua perfuração (Aoki et al., 2018; Wang et al., 2019), ou atua como um "seguro de membrana" para possivelmente selar qualquer perfuração não detectada (Toffler et al., 2010; Wang et al., 2019); ou seja, parece ser capaz de tratar a perfuração da membrana sinusal e permitir que a cirurgia seja concluída (Choukroun 
et al., 2006). A PRF funciona dessa forma pois serve como um amortecedor abaixo do assoalho do seio maxilar, protegendo a membrana (Narang et al., 2015).

A utilização de PRF como único material para a estimulação de neoformação óssea e cicatrização tecidual tem resultados promissores. É uma técnica simples, sendo um método fácil e bem-sucedido para cobrir a membrana sinusal ou a janela da osteotomia (Ali et al., 2015; Damsaz et al., 2020; Dominiak et al., 2021).) além de ser segura e econômica (Molemans et al., 2019). Quando avaliada comparativamente a formação óssea entre a utilização da PRF sozinha e o coágulo sanguíneo no preenchimento na cirurgia de elevação do seio maxilar, não houve diferença estatisticamente significante entre esses dois grupos. No entanto, aumentou o ganho ósseo nas regiões mesial, bucal e palatal, além de aumento da altura média e largura buco-palatina do seio maxilar antes e depois da cirurgia. Assim, observou-se que a PRF pode ser mais eficaz como um único material de preenchimento sinusal (Kaarthikeyan, Jayakumar \& Sivakumar, 2019) além de fornecer resultados favoráveis na elevação do seio quando a AORM é baixa (Aoki et al., 2018). Estudos futuros sobre a altura do osso alveolar vertical após o uso de PRF como único material de preenchimento para levantamento do seio maxilar são recomendados (Ocak et al., 2017).

A PRF pode ser adicionada também aos enxertos nos procedimentos cirúrgicos. A sua adição ao enxerto alógeno de osso liofilizado desmineralizado acelera a maturação do enxerto e diminui o período de cicatrização antes da colocação do implante (Ali et al., 2015; Barbu et al., 2018; Narang et al., 2015 e Pichotano et al., 2019) e é um material confiável (Kumar et al., 2018). A quantidade de material ósseo utilizada para preencher a cavidade sinusal pode ser reduzida com segurança, sem prejudicar a densidade óssea final (Choukroun et al., 2006). Isso pôde ser observado na avaliação histológica conduzida por Chokroun et al. (2006), onde foi comparado o levantamento de assoalho de seio maxilar usando PRF com partículas de aloenxerto liofilizado (AOL) e o AOL sem o PRF, revelando, a idêntica maturação histológica do grupo teste em 4 meses comparado ao grupo controle em 8 meses (Choukroun et al., 2006). Esse tempo também foi observado no estudo clínico com instalação tardia de implantes após levantamento sinusal, onde a adição de L-PRF ao enxerto MOBD aumentou o osso recém-formado após 4 meses de cicatrização, enquanto o MOBD sozinho (grupo controle) levou 8 meses (Pichotano et al., 2019). Quando L-PRF foi adicionada aos xenoenxertos mais comuns, histologicamente, a cicatrização óssea também foi mais rápida (Castro et al., 2017). Em um estudo em ovelhas, observando os resultados histológicos, observou-se que osso autógeno e mistura de osso bovino representou um potencial de regeneração superior do que o enxerto de apenas membrana PRF de seio maxilar (Ocak et al., 2017).

Apesar de resultados promissores, alguns estudos demonstraram que o uso de PRF com outros biomateriais de enxerto pareceu não fornecer efeitos benéficos ou melhorar os resultados nos procedimentos de elevação do seio (Orteja-Mejia et al., 2020). Essa informação está de acordo com a metanálise realizada por Liu et al. (2019) onde o uso de PRF como material auxiliar para enxerto ósseo na ampliação do seio não é atualmente recomendado para uso rotineiro devido às evidências limitadas. Os autores concluíram que as técnicas de preparação da PRF foram diferentes nos estudos incluídos, contribuindo com um grande viés. Embora a adição de PRF aos substitutos ósseos possa ajudar a reduzir o tempo de cicatrização, seu uso como material auxiliar não parece realmente melhorar a efetividade do aumento do seio (Liu et al., 2019). Na revisão sistemática de Dragonas et al. (2018), o uso de L-PRF em procedimentos de aumento do seio maxilar também não foi associado a resultados mais favoráveis. Para Strauss, Stähli e Gruber (2018) os resultados dos procedimentos de elevação do assoalho sinusal utilizando a PRF são inconclusivos e o efeito da PRF na regeneração óssea durante a elevação do assoalho do seio permanece questionável.

Mesmo que as membranas de L-PRF sejam particularmente adaptadas à prática diária de cirurgia periodontal, a relevância dessa técnica a longo prazo depende muito da capacidade dos pesquisadores em definir com precisão as melhores indicações, selecionar o tipo e a quantidade adequados de material ósseo a combinar com a L-PRF (Del Corso et al., 2012). A comunidade clínica exige uma padronização dos protocolos de PRF para examinar melhor o benefício da PRF na regeneração de ossos e tecidos moles em estudos reprodutíveis, com um nível científico mais alto de evidência (Damsaz et al., 2020; Ghanaati et al., 2018). A literatura atual não permite tirar conclusões definitivas sobre os vários PRPs e L-PRF e a melhor maneira de usá- 
los para a cicatrização óssea em cirurgia oral e maxilofacial. É necessário acompanhamento a longo prazo e uma amostra grande para confirmar a previsibilidade desse procedimento. As evidências limitadas sobre os efeitos da L-PRF nos procedimentos de enxerto ósseo intraoral destacam a necessidade de mais pesquisas para avaliar completamente suas indicações clínicas (Dragonas et al., 2018; Strauss, Stähli \& Gruber, 2018). Ensaios clínicos randomizados bem projetados, com acompanhamento de longo prazo, incluindo o mesmo protocolo de obtenção da PRF, são necessários.

\section{Considerações Finais}

De A PRF é um biomaterial autólogo utilizado para a cicatrização que incorpora em uma matriz de fibrina autóloga, leucócitos, plaquetas, citocinas leucocitárias e fatores de crescimento. É um método fácil, simples, econômico e bem-sucedido de auxílio na reparação tecidual, tanto de tecidos moles quanto duros, na cirurgia de levantamento de seio maxilar, além de atuar como uma barreira diminuindo os riscos de perfuração da membrana sinusal. No entanto, ainda não existe um consenso em relação à sua contribuição efetiva na cirurgia de levantamento de seio.

\section{Referências}

Agrawal A. A. (2017). Evolution, current status and advances in application of platelet concentrate in periodontics and implantology. World journal of clinical cases, 5(5), 159-171.

Ali, S., Bakry, S. A., \& Abd-Elhakam, H. (2015). Platelet-Rich Fibrin in Maxillary Sinus Augmentation: A Systematic Review. The Journal of oral implantology, 41(6), 746-753.

Aoki, N., Maeda, M., Kurata, M., Hirose, M., Ojima, Y., Wada, K., \& Shibuya, Y. (2018). Sinus floor elevation with platelet-rich fibrin alone: A Clinical retrospective study of 1-7 years. Journal of clinical and experimental dentistry, 10(10), e984-e991.

Barbu, H. M., Andreescu, C. F., Comaneanu, M. R., Referendaru, D., \& Mijiritsky, E. (2018). Maxillary Sinus Floor Augmentation to Enable One-Stage Implant Placement by Using Bovine Bone Substitute and Platelet-Rich Fibrin. BioMed research international, $2018,6562958$.

Castro, A. B., Meschi, N., Temmerman, A., Pinto, N., Lambrechts, P., Teughels, W., \& Quirynen, M. (2017). Regenerative potential of leucocyte- and plateletrich fibrin. Part B: sinus floor elevation, alveolar ridge preservation and implant therapy. A systematic review. Journal of clinical periodontology, 44(2), 225234.

Choukroun, J., Diss, A., Simonpieri, A., Girard, M. O., Schoeffler, C., Dohan, S. L., Dohan, A. J., Mouhyi, J., \& Dohan, D. M. (2006). Platelet-rich fibrin (PRF): a second-generation platelet concentrate. Part V: histologic evaluations of PRF effects on bone allograft maturation in sinus lift. Oral surgery, oral medicine, oral pathology, oral radiology, and endodontics, 101(3), 299-303.

Damsaz, M., Castagnoli, C. Z., Eshghpour, M., Alamdari, D. H., Alamdari, A. H., Noujeim, Z., \& Haidar, Z. S. (2020). Evidence-Based Clinical Efficacy of Leukocyte and Platelet-Rich Fibrin in Maxillary Sinus Floor Lift, Graft and Surgical Augmentation Procedures. Frontiers in surgery, 7, 537138.

Del Corso, M., Vervelle, A., Simonpieri, A., Jimbo, R., Inchingolo, F., Sammartino, G., \& Dohan Ehrenfest, D. M. (2012). Current knowledge and perspectives for the use of platelet-rich plasma (PRP) and platelet-rich fibrin (PRF) in oral and maxillofacial surgery part 1: Periodontal and dentoalveolar surgery. Current pharmaceutical biotechnology, 13(7), 1207-1230.

Dominiak, S., Karuga-Kuźniewska, E., Popecki, P., \& Kubasiewicz-Ross, P. (2021). PRF versus xenograft in sinus augmentation in case of HA-coating implant placement: A 36-months retrospective study. Advances in clinical and experimental medicine: official organ Wroclaw Medical University, $10.17219 /$ acem/134202.

Dragonas, P., Katsaros, T., Avila-Ortiz, G., Chambrone, L., Schiavo, J. H., \& Palaiologou, A. (2019). Effects of leukocyte-platelet-rich fibrin (L-PRF) in different intraoral bone grafting procedures: a systematic review. International journal of oral and maxillofacial surgery, 48(2), $250-262$.

Ghanaati, S., Herrera-Vizcaino, C., Al-Maawi, S., Lorenz, J., Miron, R. J., Nelson, K., Schwarz, F., Choukroun, J., \& Sader, R. (2018). Fifteen Years of Platelet Rich Fibrin in Dentistry and Oromaxillofacial Surgery: How High is the Level of Scientific Evidence?. The Journal of oral implantology, 44(6), 471-492.

Kaarthikeyan, G., Jayakumar, N. D., \& Sivakumar, D. (2019). Comparative Evaluation of Bone Formation between PRF and Blood Clot Alone as the Sole Sinus-Filling Material in Maxillary Sinus Augmentation with the Implant as a Tent Pole: A Randomized Split-Mouth Study. Journal of long-term effects of medical implants, 29(2), 105-111.

Kumar, K. R., Genmorgan, K., Abdul Rahman, S. M., Rajan, M. A., Kumar, T. A., \& Prasad, V. S. (2016). Role of plasma-rich fibrin in oral surgery. Journal of pharmacy \& bioallied sciences, 8(Suppl 1), S36-S38.

Kumar, M., Chopra, S., Das, D., Gupta, M., Memoalia, J., \& Verma, G. (2018). Direct Maxillary Sinus Floor Augmentation for Simultaneous Dental Implant Placement. Annals of maxillofacial surgery, 8(2), 188-192.

Liu, Z., Li, C., Zhou, J., Sun, X., Li, X., Qi, M., \& Zhou, Y. (2018). Endoscopically controlled flapless transcrestal sinus floor elevation with platelet-rich fibrin followed by simultaneous dental implant placement: A case report and literature review. Medicine, 17(97). 
Liu, R., Yan, M., Chen, S., Huang, W., Wu, D., \& Chen, J. (2019). Effectiveness of Platelet-Rich Fibrin as an Adjunctive Material to Bone Graft in Maxillary Sinus Augmentation: A Meta-Analysis of Randomized Controlled Trails. BioMed research international, 2019, 7267062.

Molemans, B., Cortellini, S., Jacobs, R., Pinto, N., Teughels, W., \& Quirynen, M. (2019). Simultaneous sinus floor elevation and implant placement using leukocyte- and platelet-rich fibrin as a sole graft material. The International journal of oral \& maxillofacial implants, 34(5), 1195-1201.

Narang, S., Parihar, A. S., Narang, A., Arora, S., Katoch, V., \& Bhatia, V. (2015). Modified osteotome sinus floor elevation using combination platelet rich fibrin, bone graft materials, and immediate implant placement in the posterior maxilla. Journal of Indian Society of Periodontology, 19(4), 462-465.

Ocak, H., Kutuk, N., Demetoglu, U., Balcıglu, E., Ozdamar, S., \& Alkan, A. (2017). Comparison of Bovine Bone-Autogenic Bone Mixture Versus PlateletRich Fibrin for Maxillary Sinus Grafting: Histologic and Histomorphologic Study. The Journal of oral implantology, 43(3), 194-201.

Ortega-Mejia, H., Estrugo-Devesa, A., Saka-Herrán, C., Ayuso-Montero, R., López-López, J., \& Velasco-Ortega, E. (2020). Platelet-Rich Plasma in Maxillary Sinus Augmentation: Systematic Review. Materials (Basel, Switzerland), 13(3), 622.

Pichotano, E. C., de Molon, R. S., de Souza, R. V., Austin, R. S., Marcantonio, E., \& Zandim-Barcelos, D. L. (2019). Evaluation of L-PRF combined with deproteinized bovine bone mineral for early implant placement after maxillary sinus augmentation: A randomized clinical trial. Clinical implant dentistry and related research, 21(2), 253-262.

Shah, R., M G, T., Thomas, R., \& Mehta, D. S. (2017). An Update on the Protocols and Biologic Actions of Platelet Rich Fibrin in Dentistry. The European journal of prosthodontics and restorative dentistry, 25(2), 64-72.

Simonpieri, A., Del Corso, M., Vervelle, A., Jimbo, R., Inchingolo, F., Sammartino, G., \& Dohan Ehrenfest, D. M. (2012). Current knowledge and perspectives for the use of platelet-rich plasma (PRP) and platelet-rich fibrin (PRF) in oral and maxillofacial surgery part 2: Bone graft, implant and reconstructive surgery. Current pharmaceutical biotechnology, 13(7), 1231-1256.

Strauss, F. J., Stähli, A., \& Gruber, R. (2018). The use of platelet-rich fibrin to enhance the outcomes of implant therapy: A systematic review. Clinical oral implants research, 29 Suppl 18(Suppl Suppl 18), 6-19.

Testori, T., Panda, S., Clauser, T., Scaini, R., Zuffetti, F., Capelli, M., Taschieri, S., Mortellaro, C., \& Del Fabbro, M. (2019). Short implants and platelet-rich fibrin for transcrestal sinus floor elevation: a prospective multicenter clinical study. Journal of biological regulators and homeostatic agents, $33(6$ Suppl. 2), $121-135$.

Toffler, M., Toscano, N., \& Holtzclaw, D. (2010). Osteotome-mediated sinus floor elevation using only platelet-rich fibrin: an early report on 110 patients. Implant dentistry, 19(5), 447-456.

Wang, H., Wang, J., Guo, T., Ding, X., Yu, W., Zhao, J., \& Zhou, Y. (2019). The endoscopically assisted transcrestal sinus floor elevation with platelet-rich fibrin at an immediate implantation of periapical lesion site: A case report. Medicine, 98(27), e16251. 